\title{
Optimal Control of a Viscous Shallow Water Equation
}

\author{
Wenbin Zhang $^{1}$ and Jiangbo Zhou $^{2}$ \\ ${ }^{1}$ Taizhou Institute of Science and Technology (NUST) Taizhou, Jiangsu 225300, China \\ ${ }^{2}$ Nonlinear Scientific Research Center, Faculty of Science, Jiangsu University, Zhenjiang, Jiangsu 212013, China
}

Correspondence should be addressed to Wenbin Zhang; zwbnust@126.com

Received 22 June 2013; Accepted 23 August 2013

Academic Editor: Shao-Ming Fei

Copyright (C) 2013 W. Zhang and J. Zhou. This is an open access article distributed under the Creative Commons Attribution License, which permits unrestricted use, distribution, and reproduction in any medium, provided the original work is properly cited.

This paper is concerned with a viscous shallow water equation, which includes both the viscous Camassa-Holm equation and the viscous Degasperis-Procesi equation as its special cases. The optimal control under boundary conditions is given, and the existence of optimal solution to the equation is proved.

\section{Introduction}

Holm and Staley [1] studied the following family of evolutionary 1+1 PDEs:

$$
m_{t}+\underbrace{u_{x} m}_{\text {convection }}+b \underbrace{u m_{x}}_{\text {stretching }}=\varepsilon \underbrace{m_{x x}}_{\text {viscosity }} \text {, }
$$

which describes the balance between convection and stretching for small viscosity in the dynamics of one-dimensional nonlinear waves in fluids. Here $u=p * m=\int_{-\infty}^{\infty} p(x-$ $y) m(y) d y, m=u-u_{x x}$, and $p$ is chosen to be the Green's function for the Helmholtz operator $1-\partial_{x}^{2}$ on the line. In a recent study of soliton equations, it is found that (1) for $\varepsilon=0$ and any $b \neq-1$ is included in the family of shallow water equations at quadratic order accuracy that are asymptotically equivalent under Kodama transformations [2].

When $\varepsilon=0$, (1) becomes the $b$-family of equations:

$$
m_{t}+u_{x} m+b u m_{x}=0
$$

which describes a one-dimensional version of active fluid transport. It was shown by Degasperis and Procesi [3] that (2) cannot satisfy the asymptotic integrability condition unless $b=2$ or $b=3$; compare $[2,4,5]$.

For $b=2$ in (2), it becomes the Camassa-Holm $(\mathrm{CH})$ equation:

$$
u_{t}-u_{x x t}+3 u u_{x}=2 u_{x} u_{x x}+u u_{x x x}
$$

which is a model describing the unidirectional propagation of shallow water waves over a flat bottom [4]. Equation (3) has a bi-Hamiltonian structure [6] and is completely integrable $[7,8]$. It admits, in addition to smooth waves, a multitude of traveling wave solutions with singularities: peakons, cuspons, stumpons, and composite waves $[4,9]$. Its solitary waves are stable solitons $[10,11]$, retaining their shape and form after interactions [10]. The Cauchy problem of (3) has been studied extensively. Constantin [12] and Rodríguez-Blanco [13] investigated the locally well-posed for initial data $u_{0} \in$ $H^{s}(R)$ with $s>3 / 2$. More interestingly, it has strong solutions that are global in time $[11,14]$ as well as solutions that blow up in finite time $[11,15,16]$. On the other hand, Bressan and Constantin [17] and Xin and Zhang [18] showed that (3) has global weak solutions with initial data $u_{0} \in H^{1}$.

For $b=3$ in (2), it becomes the Degasperis-Procesi (DP) equation:

$$
u_{t}-u_{x x t}+4 u u_{x}=3 u_{x} u_{x x}+u u_{x x x},
$$

which can be used as a model for nonlinear shallow water dynamics, and its asymptotic accuracy is the same as (3). Degasperis et al. [5] presented that (4) has a bi-Hamiltonian structure with an infinite sequence of conserved quantities and admits exact peakon solutions which are analogous to (3) peakons $[4,10,19]$. Dullin et al. [20] showed that (4) can be obtained from the shallow water elevation equation by an appropriate Kodama transformation. The numerical stability of solitons and peakons, the multisoliton solutions 
and their peakon limits, and an inverse scattering method to compute N-peakon solutions to (4) have been investigated, respectively, in [21-23]. After (4) appeared, it has attracted many researchers to discover its dynamics (see [24-31]). Yin $[24,25]$ proved the local well-posedness of (4) with initial data $u_{0} \in H^{s}(R)(s>3 / 2)$ on the line and on the circle and derived the precise blow-up scenario and a blow-up result. The global existence of strong solutions and global weak solutions to (4) were studied in $[29,30]$. Similar to (3) $[11,15$, $18,22-24]$, (4) has not only global strong solutions [27, 28] but also blow-up solutions [26, 28-30]. Apart from these, Coclite and Karlsen [31] proved that it has global entropy weak solutions in $L^{2}(R) \cap B V(R)$ and $L^{2}(R) \cap L^{4}(R)$.

Recently, Lai and Wu [32] found that, in both models (1) and (2), the coefficient of $u u_{x}$ is equal to the coefficient of $u_{x} u_{x x}$ plus the one of $u u_{x x x}$. That is,

$$
3=2+1, \quad 4=3+1 .
$$

Then, they studied the global solutions and blow-up phenomena to the following generalized equation:

$$
u_{t}-u_{x x t}+(a+b) u u_{x}=a u_{x} u_{x x}+b u u_{x x x} .
$$

In this paper, we study the optimal control problem for the following equation:

$$
u_{t}-u_{x x t}-\varepsilon\left(u_{x x}-u_{x x x x}\right)+(a+b) u u_{x}=a u_{x} u_{x x}+b u u_{x x x} \text {, }
$$

where $a, b$ are positive constants. The optimal control is an important component of modern control theories and has a wider application in modern engineering. Two methods are introduced to study the control problems in PDE: one is using a low model method and then changing to an ODE model [33]; the other is using a quasi-optimal control method [34]. No matter which one is chosen, it is necessary to prove the existence of optimal solution according to the basic theory [35]. The control problems of nonlinear PDE have been studied extensively. Kunisch and Volkwein solved open-loop and closed-loop optimal control problems for the Burgers equation [36] and discussed the instantaneous control of the equation [37]. Vedantham [38] developed a technique to utilize the Cole-Hopf transformation to solve an optimal control problem for the Burgers equation. Øksendal [39] proved a sufficient maximum principle for the optimal control systems described by a quasi-linear stochastic heat equation. In [40], Ghattas and Bark studied the optimal control of two- and three-dimensional incompressible NavierStokes flows. Lagnese and Leugering [41] considered the problem of boundary optimal control of a wave equation with boundary dissipation. In [42], Yong established a unified existence theory of optimal controls for general semilinear evolutionary distributed parameter systems under the framework of mild (or weak) solutions for evolution equations. Yong and Zheng [43] considered the Cahn-Hilliard equation in a bounded domain with smooth boundary. Based on the energy estimates and the compact method, Ryu and Yagi $[44,45]$ studied Keller-Segel equations and adsorbateinduced phase transition model. Tian et al. [46-48] studied the optimal control problems for parabolic equations, such as viscous $\mathrm{CH}$ equation, viscous DP equation, and viscous DGH equation. Under boundary condition, Zhao and Liu [49] studied viscous Cahn-Hilliard equation.

With $m=u-u_{x x}$, the optimal control problem for (7) we intend to investigate is

$$
\min J(m, \omega)=\frac{1}{2}\|C m-z\|_{S}^{2}+\frac{\delta}{2}\|\omega\|_{L^{2}\left(Q_{0}\right)}^{2},
$$

subject to

$$
\begin{gathered}
m_{t}-\varepsilon m_{x x}+a u_{x} m+b_{x}=B^{*} \omega \quad \text { in }(0, T) \times \Omega, \\
u=u_{x}=u_{x x}=0 \quad \text { on }(0, T) \times \partial \Omega, \\
m(0, x)=m_{0}(x) \quad \text { in } \Omega,
\end{gathered}
$$

where $\Omega=(0,1)$. Clearly, our control target is to match the given desired state $z$ by adjusting the body force $\omega$ in a control volume $Q_{0} \subseteq Q=(0, T) \times \Omega$ in the $L^{2}$-sense.

Notation. In this paper, we set that $V=H_{0}^{1}(0,1), H=L^{2}(0$, $1), V^{*}=H^{-1}(0,1)$, and $H^{*}=L^{2}(0,1)$ are dual space, respectively. Then, $V \hookrightarrow H=H^{*} \hookrightarrow V^{*}$, with each embedding being dense. The extension operator $B^{*} \in \mathscr{L}\left(L^{2}\left(Q_{0}\right), L^{2}\left(V^{*}\right)\right)$ is given by

$$
B^{*} q= \begin{cases}q, & q \in Q_{0} \\ 0, & q \in \frac{Q}{Q_{0}} .\end{cases}
$$

We supply the Lebesgue space $L^{2}$ with the norm $\|\cdot\|$ and the Sobolev space $H^{s}, s \in R$ with the norm $\|\cdot\|_{H^{s}}$. For a fixed $T>0$, we also defined a space $W(0, T ; V)$ as

$$
W(0, T ; V)=\left\{m: m \in L^{2}(0, T ; V), m_{t} \in L^{2}\left(0, T ; V^{*}\right)\right\},
$$

which is a Hilbert space endowed with common inner product.

\section{Existence of Unique Weak Solution}

In this section, we prove the existence of a weak solution for the following equation:

$$
m_{t}-\varepsilon m_{x x}+a u_{x} m+b u m_{x}=B^{*} \omega \quad \text { in }(0, T) \times \Omega,
$$

with the boundary conditions

$$
u=u_{x}=u_{x x}=0 \quad \text { on }(0, T) \times \partial \Omega,
$$

and the initial value

$$
m(x, 0)=m_{0}(x) \quad \text { in } \Omega,
$$

where $m=u-u_{x x}, \Omega=(0,1), B^{*} \omega \in L^{2}\left(V^{*}\right)$, and a control $\omega \in L^{2}\left(Q_{0}\right)$. Now, we give the definition of the weak solution in the space $W(0, T ; V)$. 
Definition 1. A function $m(x, t) \in W(0, T ; V)$ is called a weak solution to (12), if

$$
\begin{aligned}
& \frac{d}{d t}(m, \phi)+\varepsilon\left(m_{x}, \phi_{x}\right)+a\left(u_{x} m, \phi\right)+b\left(u m_{x}, \phi\right) \\
& =\left(B^{*} \omega, \phi\right)_{V^{*}, V},
\end{aligned}
$$

is valid for all $\phi \in V$, a.e. $t \in[0, T]$ and $m_{0}(x) \in H$.

By using the standard Galerkin method and some a priori estimates, one can obtain the following theorem, which ensures the existence of a unique weak solution to the viscous shallow water equation.

Theorem 2. Let $m_{0}(x) \in H$ and $B^{*} \omega \in L^{2}\left(V^{*}\right)$ then the problem (12)-(14) admits a local weak solution $m(x, t)$ which satisfies Definition 1.

Proof. Let $\left\{\varphi_{i}\right\}_{i=1}^{\infty}$ be an orthonormal basis in the space $H$ consisting of eigenfunctions of the operator $A=-\partial_{x}^{2}$. For $k \epsilon$ $\mathcal{N}$, we define the discrete ansatz space by $V_{k}=\operatorname{span}\left\{\varphi_{1}, \varphi_{2}\right.$, $\left.\ldots, \varphi_{k}\right\} \subset V$. Let $m_{k}(t)=m_{k}(x, t)=\sum_{i=1}^{k} m_{i}^{k}(t) \varphi_{i}(x)$ with $m_{k}(\cdot, 0) \rightarrow m_{0}$ in $H$.

By analyzing the limiting behavior of the sequences of smooth functions $\left\{u_{k}\right\}$ and $\left\{m_{k}\right\}$, we can prove the existence of a weak solution to (12).

Performing the Galerkin procedure for the problem (12)(14), we obtain

$$
\begin{gathered}
m_{k, t}-\varepsilon m_{k, x x}+a u_{k, x} m_{k}+b u_{k} m_{k, x} \\
=B^{*} \omega \text { in }(0, T) \times \Omega,
\end{gathered}
$$

with the boundary conditions

$$
u_{k}=u_{k, x}=u_{k, x x}=0 \quad \text { on }(0, T) \times \partial \Omega,
$$

and the initial value

$$
m_{k}(x, 0)=m_{k, 0}(x) \in H \quad \text { in } \Omega .
$$

Clearly, (16) is an ordinary differential equation, and, according to standard ODE's theory, there is a unique solution to $(16)$ in the interval $\left[0, t_{k}\right)$. What we should do is to show that the solution is uniformly bounded as $t_{k} \rightarrow T$. We will prove the existence of weak solution in the following steps.

Step 1. Taking the inner product of (16) with $u_{k}$ in $\Omega$, we have

$$
\begin{gathered}
\frac{1}{2} \frac{d}{d t}\left(\left\|u_{k}\right\|^{2}+\left\|u_{k, x}\right\|^{2}\right)+\varepsilon\left(\left\|u_{k, x}\right\|^{2}+\left\|u_{k, x x}\right\|^{2}\right) \\
=-\frac{a-2 b}{2}\left(u_{k, x}, u_{k, x}^{2}\right)+\left(B^{*} \omega, u_{k}\right)_{V^{*}, V} .
\end{gathered}
$$

Let us estimate the first term of the right hand side of (19) as follows:

$$
\left|-\frac{a-2 b}{2}\left(u_{k, x}, u_{k, x}^{2}\right)\right| \leq \frac{|a-2 b|}{2}\left\|u_{k, x}\right\|_{L^{\infty}}\left\|u_{k, x}\right\|^{2} .
$$

By the Sobolev embedding theorem and the Poincare inequality, we obtain

$$
\left\|u_{k, x}\right\|_{L^{\infty}} \leq c_{1}\left\|u_{k, x}\right\|_{H^{1}} \leq c_{1}\left\|u_{k}\right\|_{H^{2}} \leq c_{2}\left\|u_{k, x x}\right\|,
$$

where $c_{1}, c_{2}$ are constants.

It follows from (20) and (21) that

$$
\left|-\frac{a-2 b}{2}\left(u_{k, x}, u_{k, x}^{2}\right)\right| \leq \frac{|a-2 b|}{2} c_{2}\left\|u_{k, x x}\right\| \cdot\left\|u_{k, x}\right\|^{2} .
$$

Next, we estimate the second term of the right hand side of (19). Noting that $B^{*} \omega \in L^{2}\left(V^{*}\right)$ is a control item, we can assume that $\left\|B^{*} \Phi\right\|_{V^{*}} \leq M$, where $M$ is positive constant. Then

$$
\left|\left(B^{*} \varpi, u_{k}\right)_{V^{*}, V}\right| \leq\left\|B^{*} \varpi\right\|_{V^{*}}\left\|u_{k}\right\|_{V} \leq c_{3} M\left\|u_{k, x}\right\| .
$$

Combining inequalities (22) and (23) with (19) and using the Young inequality, we have

$$
\begin{aligned}
& \frac{1}{2} \frac{d}{d t}\left(\left\|u_{k}\right\|^{2}+\left\|u_{k, x}\right\|^{2}\right)+\varepsilon\left(\left\|u_{k, x}\right\|^{2}+\left\|u_{k, x x}\right\|^{2}\right) \\
& \leq \frac{|a-2 b|}{2} c_{2}\left\|u_{k, x x}\right\| \cdot\left\|u_{k, x}\right\|^{2}+c_{3} M\left\|u_{k, x}\right\| \\
& \leq \varepsilon\left\|u_{k, x x}\right\|^{2}+\frac{(a-2 b)^{2} c_{2}^{2}}{4 \varepsilon}\left\|u_{k, x}\right\|^{4}+\varepsilon\left\|u_{k}\right\|^{2}+\frac{c_{3}^{2} M^{2}}{\varepsilon} \\
& \leq \varepsilon\left(\left\|u_{k, x x}\right\|^{2}+\left\|u_{k}\right\|^{2}\right)+\frac{(a-2 b)^{2} c_{2}^{2}}{4 \varepsilon}\left(\left\|u_{k}\right\|^{2}+\left\|u_{k, x}\right\|^{2}\right)^{2} \\
& +\frac{c_{3}{ }^{2} M^{2}}{\varepsilon} .
\end{aligned}
$$

Therefore, we obtain

$$
\begin{aligned}
& \frac{d}{d t}\left(\left\|u_{k}\right\|^{2}+\left\|u_{k, x}\right\|^{2}\right) \\
& \quad \leq \frac{(a-2 b)^{2} c_{2}^{2}}{2 \varepsilon}\left(\left\|u_{k}\right\|^{2}+\left\|u_{k, x}\right\|^{2}\right)^{2}+\frac{2 c_{3}{ }^{2} M^{2}}{\varepsilon} .
\end{aligned}
$$

It, thus, transpires that

$$
\begin{aligned}
& \left\|u_{k}\right\|^{2}+\left\|u_{k, x}\right\|^{2} \\
& \quad \leq \frac{2 c_{2} M}{|a-2 b| c_{2}} \tan \left\{\frac{c_{2} c_{3} M|a-2 b|}{\varepsilon} t+c_{4}\right\} \triangleq \rho_{1}^{2},
\end{aligned}
$$

where $t \in[0, T], T<\pi \varepsilon / 2 c_{2} c_{3} M|a-2 b|$ and $c_{4}=\arctan (\mid a-$ $\left.2 b \mid c_{2}\left(\left\|u_{k, 0}\right\|^{2}+\left\|u_{k, x}(0)\right\|^{2}\right) / 2 c_{3} M\right)$.

From the above discussion, we know that $\left\|u_{k}\right\| \leq \rho_{1}$ and $\left\|u_{k, x}\right\| \leq \rho_{1}$. By the Sobolev embedding theorem, we obtain

$$
\left\|u_{k}\right\|_{L^{\infty}} \leq\left\|u_{k}\right\|_{H^{1}}=\left\|u_{k}\right\|_{V} \leq\left(\left\|u_{k}\right\|+\left\|u_{k, x}\right\|\right) \leq c_{5} .
$$

Step 2. We prove a uniform $L^{2}(0, T ; V)$ bound on a sequence $\left\{m_{k}\right\}$. Taking the inner product of (16) with $m_{k}$ in $\Omega$, we have

$$
\begin{aligned}
\frac{1}{2}\left\|m_{k}\right\|^{2}+\varepsilon\left\|m_{k, x}\right\|^{2}= & (2 a-b)\left(u_{k}, m_{k} m_{k, x}\right) \\
& +\left(B^{*} \omega, m_{k}\right)_{V^{*}, V} .
\end{aligned}
$$


Let us estimate the first term of the right hand side of (28). By the Poincaré inequality and the Hölder inequality, we have

$$
\begin{aligned}
& \left|(2 a-b)\left(u_{k}, m_{k} m_{k, x}\right)\right| \\
& \quad \leq|2 a-b|\left\|u_{k}\right\|_{L^{\infty}}\left\|m_{k}\right\| \cdot\left\|m_{k, x}\right\| \\
& \quad \leq \frac{c_{5}|2 a-b|}{2}\left(\left\|m_{k}\right\|^{2}+\left\|m_{k, x}\right\|^{2}\right) \\
& \quad \leq \frac{c_{5}|2 a-b|}{2}\left(c_{6}\left\|m_{k, x}\right\|^{2}+\left\|m_{k, x}\right\|^{2}\right) \\
& \quad=c_{7}\left\|m_{k, x}\right\|^{2},
\end{aligned}
$$

where $c_{7}=\left(c_{5}|2 a-b| / 2\right)\left(c_{6}+1\right)$.

Furthermore, we estimate the second term of the right hand side of (28) in the following way:

$$
\left|\left(B^{*} \omega, m_{k}\right)_{V^{*}, V}\right| \leq M\left\|m_{k}\right\|_{V} \leq c_{8} M\left\|m_{k, x}\right\| .
$$

Combining inequalities (29) and (30) with (28), we have

$$
\frac{1}{2} \frac{d}{d t}\left\|m_{k}\right\|^{2}+\varepsilon\left\|m_{k, x}\right\|^{2} \leq c_{7}\left\|m_{k, x}\right\|^{2}+c_{8} M\left\|m_{k, x}\right\|,
$$

where $\varepsilon>c_{7}$. It follows from (31) with the Young inequality that

$$
\frac{d}{d t}\left\|m_{k}\right\|^{2}+\left(\varepsilon-c_{7}\right)\left\|m_{k, x}\right\|^{2} \leq \frac{c_{8}{ }^{2} M^{2}}{\varepsilon-c_{7}} .
$$

Integrating the pervious inequality with respect to $t$ on $[0, T]$, we obtain

$$
\int_{0}^{T}\left\|m_{k, x}\right\|^{2} d t \leq \frac{1}{\varepsilon-c_{7}}\left[\frac{c_{8}{ }^{2} M^{2} T}{\varepsilon-c_{7}}+\left\|m_{k, 0}\right\|^{2}\right] \triangleq \rho_{2}^{2} .
$$

Furthermore, we have

$$
\left\|m_{k}\right\|_{L^{2}(0, T ; V)}^{2}=\int_{0}^{T}\left\|m_{k}\right\|_{H^{1}}^{2} d t \leq c_{8} \int_{0}^{T}\left\|m_{k, x}\right\|^{2} d t \triangleq \rho_{3}^{2} .
$$

From (32), we also have

$$
\frac{d}{d t}\left\|m_{k}\right\|^{2} \leq \frac{c_{8}{ }^{2} M^{2}}{\varepsilon-c_{7}} .
$$

Integrating the pervious inequality with respect to $t<T$ on $[0, t]$, we obtain

$$
\left\|m_{k}\right\|^{2} \leq \frac{c_{8}{ }^{2} M^{2} t}{\varepsilon-c_{7}}+\left\|m_{k, 0}\right\|^{2} \triangleq \rho_{4}^{2}, \quad \forall t \in[0, T] .
$$

Step 3. We prove a uniform $L^{2}\left(0, T ; V^{*}\right)$ bound on a sequence $\left\{m_{k, t}\right\}$. By (16) and the Sobolev embedding theorem, we have

$$
\begin{aligned}
\left\|m_{k, t}\right\|_{V^{*}} \leq & \varepsilon\left\|m_{k}\right\|_{V}+a\left\|u_{k}\right\|\left\|m_{k}\right\|_{V} \\
& +b\left\|u_{k}\right\|_{V}\left\|m_{k}\right\|+\left\|B^{*} \omega\right\|_{V^{*}} \\
\leq & \varepsilon\left\|m_{k}\right\|_{V}+a \rho_{1}\left\|m_{k}\right\|_{V}+b c_{5}\left\|m_{k}\right\|+M .
\end{aligned}
$$

Furthermore, we have

$$
\left\|m_{k, t}\right\|_{V^{*}}^{2} \leq 3\left(\varepsilon+a \rho_{1}\right)^{2}\left\|m_{k}\right\|_{V}^{2}+3 b^{2} c_{5}^{2}\left\|m_{k}\right\|^{2}+3 M^{2} .
$$

Integrating (38) with respect to $t$ on $[0, T]$, we deduce that

$$
\left\|m_{k, t}\right\|_{L^{2}\left(0, T ; V^{*}\right)}^{2} \leq 3\left(\varepsilon+a \rho_{1}\right)^{2} \rho_{3}^{2}+3\left(b^{2} c_{5}^{2} \rho_{4}^{2}+M^{2}\right) T \triangleq \rho_{5}^{2} .
$$

From the pervious discussion one has the following.

(a) For every $T<\pi \varepsilon / 2 c_{2} c_{3} M|a-2 b|, \rho_{3}$ and $\rho_{4}$ are two constants and the sequence $\left\{m_{k}\right\}$ is bounded in $L^{2}(0, T ; H)$ as well as in $L^{2}(0, T ; V)$, which is independent of the dimension of ansatz space $k$.

(b) For every $T<\pi \varepsilon / 2 c_{2} c_{3} M|a-2 b|$ and $\rho_{5}$ is constant, the sequence $\left\{m_{k, t}\right\}$ is bounded in $L^{2}\left(0, T ; V^{*}\right)$, which is independent of the dimension of ansatz space $k$.

Note that, (a) and (b) the above mentioned are equivalent to $\left\{m_{k}\right\} \subset W(0, T ; V)$ bounded, and $W(0, T ; V)$ is compactly embedded into $C(0, T ; H)$. Then, we conclude the convergence of a subsequence, again denoted by $\left\{m_{k}\right\}$ weak in $W(0, T ; V)$, weakstar in $L^{\infty}(0, T ; H)$, and strong in $L^{2}(0, T ; H)$ to a function $m(x, t) \in W(0, T ; V)$.

The proof of the uniqueness of the solution is similar to Theorem 1 in [37], so we omit it here. This completes the proof of the theorem.

Our next result describes that the norm of weak solution can be controlled by initial value and control item.

Theorem 3. Let $m_{0} \in H$ and $B^{*} \omega \in L^{2}\left(V^{*}\right)$; then there exists a positive constant $C$, such that

$$
\|m\|_{W(0, T ; V)}^{2} \leq C\left(\left\|m_{0}\right\|^{2}+\|\omega\|_{L^{2}\left(Q_{0}\right)}^{2}\right) .
$$

Proof. Taking the inner product of (12) with $u$ in $\Omega$, by using the same argument as in the proof of Theorem 2, we have

$$
\|u\| \leq \rho_{1}, \quad\left\|u_{x}\right\| \leq \rho_{1}, \quad\|u\|_{L^{\infty}} \leq c_{5},
$$

where $\rho_{1}$ and $c_{5}$ are positive constants as in Theorem 2. Taking the inner product of (12) with $m$ in $\Omega$, we have

$$
\frac{1}{2}\|m\|^{2}+\varepsilon\left\|m_{x}\right\|^{2}=(2 a-b)\left(u, m m_{x}\right)+\left(B^{*} \omega, m\right)_{V^{*}, V} .
$$

Let us estimate the first term of the right hand side of (42) as follows:

$$
\begin{aligned}
\left|(2 a-b)\left(u, m m_{x}\right)\right| & \leq|2 a-b|\|u\|_{L^{\infty}}\|m\| \cdot\left\|m_{x}\right\| \\
& \leq \frac{c_{5}|2 a-b|}{2}\left(\|m\|^{2}+\left\|m_{x}\right\|^{2}\right) \\
& \leq \frac{c_{5}|2 a-b|}{2}\left(c_{6}\left\|m_{x}\right\|^{2}+\left\|m_{x}\right\|^{2}\right) \\
& =c_{7}\left\|m_{x}\right\|^{2} .
\end{aligned}
$$


Furthermore, we estimate the second term of the right hand side of (42) in the following way:

$$
\left(B^{*} \omega, m\right)_{V^{*}, V} \leq\left\|B^{*} \omega\right\|_{V^{*}}\|m\|_{V} \leq c_{8}\left\|B^{*} \omega\right\|_{V^{*}}\left\|m_{x}\right\| .
$$

Combining inequalities (43) and (44) with (42), we have

$$
\frac{1}{2} \frac{d}{d t}\|m\|^{2}+\left(\varepsilon-c_{7}\right)\left\|m_{x}\right\|^{2} \leq c_{8}\left\|B^{*} \emptyset\right\|_{V^{*}}\left\|m_{x}\right\|,
$$

where $\varepsilon>c_{7}$.

Integrating (45) with respect to $t$ on $[0, T]$, we derive that

$$
\begin{gathered}
\frac{1}{2}\|m(T)\|^{2}-\frac{1}{2}\left\|m_{0}\right\|^{2}+\left(\varepsilon-c_{7}\right)\left\|m_{x}\right\|_{L^{2}(0, T ; H)}^{2} \\
\leq c_{8}\left\|B^{*} \omega\right\|_{L^{2}\left(0, T ; V^{*}\right)}\left\|m_{x}\right\|_{L^{2}(0, T ; H)} .
\end{gathered}
$$

According to the Young inequality, we have

$$
\begin{aligned}
& c_{8}\left\|B^{*} \omega\right\|_{L^{2}\left(0, T ; V^{*}\right)}\left\|m_{x}\right\|_{L^{2}(0, T ; H)} \\
& \quad \leq \frac{\left(\varepsilon-c_{7}\right)}{2}\left\|m_{x}\right\|_{L^{2}(0, T ; H)}^{2}+\frac{c_{8}{ }^{2}}{2\left(\varepsilon-c_{7}\right)}\left\|B^{*} \omega\right\|_{L^{2}\left(0, T ; V^{*}\right)}^{2} .
\end{aligned}
$$

Substituting (47) into (46) yields

$$
\left(\varepsilon-c_{7}\right)\left\|m_{x}\right\|_{L^{2}(0, T ; H)}^{2} \leq\left\|m_{0}\right\|^{2}+\frac{c_{8}{ }^{2}}{\left(\varepsilon-c_{7}\right)}\left\|B^{*} \varpi\right\|_{L^{2}\left(0, T ; V^{*}\right)}^{2} .
$$

It follows from (48) that

$$
\begin{aligned}
\|m\|_{L^{2}(0, T ; V)}^{2} & \leq c_{8} \int_{0}^{T}\left\|m_{x}\right\|^{2} d t \\
& \leq \frac{c_{8}}{\left(\varepsilon-c_{7}\right)}\left\|m_{0}\right\|^{2}+\frac{c_{8}{ }^{3}}{\left(\varepsilon-c_{7}\right)^{2}}\left\|B^{*} \omega\right\|_{L^{2}\left(0, T ; V^{*}\right)}^{2} \\
& \leq c_{9}\left(\left\|m_{0}\right\|+\left\|B^{*} \omega\right\|_{L^{2}\left(0, T ; V^{*}\right)}\right)^{2},
\end{aligned}
$$

where $c_{9}=\max \left\{c_{8} /\left(\varepsilon-c_{7}\right), c_{8}{ }^{3} /\left(\varepsilon-c_{7}\right)^{2}\right\}$.

Combining inequality (45) with $\varepsilon>c_{7}$, we also have

$$
\frac{1}{2} \frac{d}{d t}\|m\|^{2} \leq c_{8}\left\|B^{*} \omega\right\|_{V^{*}}\|m\|_{V} .
$$

Integrating the pervious inequality with respect to $t$ yields

$$
\begin{aligned}
\|m\|^{2} \leq & \left\|m_{0}\right\|^{2}+2 c_{8}\left\|B^{*} \omega\right\|_{L^{2}\left(0, T ; V^{*}\right)}\|m\|_{L^{2}(0, T ; V)} \\
\leq & \left\|m_{0}\right\|^{2}+2 c_{8}\left\|B^{*} \omega\right\|_{L^{2}\left(0, T ; V^{*}\right)} \sqrt{c_{9}} \\
& \times\left(\left\|m_{0}\right\|+\left\|B^{*} \omega\right\|_{L^{2}\left(0, T ; V^{*}\right)}\right) \\
\leq & c_{10}\left(\left\|m_{0}\right\|+\left\|B^{*} \omega\right\|_{L^{2}\left(0, T ; V^{*}\right)}\right)^{2},
\end{aligned}
$$

where $c_{10}=2 \max \left\{1, c_{8} \sqrt{c_{9}}\right\}$.
Combining (12) with the Sobolev embedding theorem, we obtain

$$
\begin{aligned}
\left\|m_{t}\right\|_{V^{*}} & \leq \varepsilon\|m\|_{V}+a\|u\|\|m\|_{V}+b\|u\|_{V}\|m\|+\left\|B^{*} \omega\right\|_{V^{*}} \\
& \leq\left(\varepsilon+a \rho_{1}\right)\|m\|_{V}+b c_{5}\|m\|+\left\|B^{*} \omega\right\|_{V^{*}} .
\end{aligned}
$$

Furthermore, we have

$$
\left\|m_{t}\right\|_{V^{*}}^{2} \leq 3\left(\varepsilon+a \rho_{1}\right)^{2}\|m\|_{V}^{2}+3 b^{2} c_{5}^{2}\|m\|^{2}+3\left\|B^{*} \omega\right\|_{V^{*}}^{2} .
$$

Integrating (53) with respect to $t$ on $[0, T]$, we deduce that

$$
\begin{aligned}
\left\|m_{t}\right\|_{L^{2}\left(0, T ; V^{*}\right)}^{2} & \\
\leq & 3\left(\varepsilon+a \rho_{1}\right)^{2}\|m\|_{L^{2}(0, T ; V)}^{2} \\
& +3 b^{2} c_{5}{ }^{2} \int_{0}^{T}\|m\|^{2} d t+3\left\|B^{*} \omega\right\|_{L^{2}\left(0, T ; V^{*}\right)}^{2} \\
\leq & 3\left(\varepsilon+a \rho_{1}\right)^{2} c_{9}\left(\left\|m_{0}\right\|+\left\|B^{*} \omega\right\|_{L^{2}\left(0, T ; V^{*}\right)}\right)^{2} \\
& +3 b^{2} c_{5}{ }^{2} c_{10} T\left(\left\|m_{0}\right\|+\left\|B^{*} \omega\right\|_{L^{2}\left(0, T ; V^{*}\right)}\right)^{2} \\
& +3\left\|B^{*} \omega\right\|_{L^{2}\left(0, T ; V^{*}\right)}^{2} \\
\leq & c_{11}\left(\left\|m_{0}\right\|+\left\|B^{*} \omega\right\|_{L^{2}\left(0, T ; V^{*}\right)}\right)^{2},
\end{aligned}
$$

where $c_{11}=3\left[\left(\varepsilon+a \rho_{1}\right)^{2} c_{9}+b^{2} c_{5}^{2} c_{10} T+1\right]$.

Taking into account (49) and (54), we arrive at

$$
\begin{aligned}
\|m\|_{W(0, T ; V)}^{2} & =\|m\|_{L^{2}(0, T ; V)}^{2}+\left\|m_{t}\right\|_{L^{2}\left(0, T ; V^{*}\right)}^{2} \\
& \leq\left(c_{9}+c_{11}\right)\left(\left\|m_{0}\right\|+\left\|B^{*} \omega\right\|_{L^{2}\left(0, T ; V^{*}\right)}\right)^{2} \\
& \leq C\left(\left\|m_{0}\right\|+\|\emptyset\|_{L^{2}\left(Q_{0}\right)}\right)^{2},
\end{aligned}
$$

where $C=c_{9}+c_{11}$. This completes the proof of the theorem.

\section{Existence of Optimal Solution to the Problem of (7)}

In this section, we consider the optimal control problem of (7) and prove the existence of optimal solution based on Lions' theory (see [50]).

Allowing a control $\varpi \in L^{2}\left(Q_{0}\right)$, we study the following control system:

$$
\begin{gathered}
m_{t}-\varepsilon m_{x x}+a u_{x} m+\text { bum }_{x}=B^{*} \emptyset \quad \text { in }(0, T) \times \Omega, \\
u=u_{x}=u_{x x}=0 \quad \text { on }(0, T) \times \partial \Omega, \\
m(x, 0)=m_{0}(x) \in H \quad \text { in } \Omega,
\end{gathered}
$$

where $m=u-u_{x x}$ and $\Omega=(0,1)$. Let $C \in L(W(0, T ; V), S)$ be an observation operator, and let $S$ be a real Hilbert space of 
observations. Then, we choose performance index of tracking type

$$
J(m, \varpi)=\frac{1}{2}\|C m-z\|_{S}^{2}+\frac{\delta}{2}\|\omega\|_{L^{2}\left(Q_{0}\right)}^{2},
$$

where $z \in S$ is a desired state and $\delta>0$ is fixed. Optimal control problem of (7) is

$$
\min J(m, \omega),
$$

where $(m, \emptyset)$ satisfies $(56)$.

Let $X=W(0, T ; V) \times L^{2}\left(Q_{0}\right), Y=L^{2}(0, T ; V) \times H$; we define an operator $e=e\left(e_{1}, e_{2}\right): X \rightarrow Y$ by

$$
\begin{gathered}
e_{1}=(-\Delta)^{-1}\left(m_{t}-\varepsilon m_{x x}+a u_{x} m+b_{u m}-B^{*} \varpi\right), \\
e_{2}=m(x, 0)-m_{0},
\end{gathered}
$$

where $\Delta$ is an operator from $V$ to $V^{*}$. Then, we rewrite (58) in the following form:

$$
\begin{array}{ll}
\min & J(m, \omega) \\
\text { s.t. } & e(m, \omega)=0 .
\end{array}
$$

Now, we give the main result.

Theorem 4. There exists an optimal control solution $\left(m^{*}, \varpi^{*}\right)$ to the problem (56).

Proof. Let $(m, \emptyset) \in X$ satisfying the equation $e(m, \emptyset)=0$. According to (57) and Theorem 3, we have

$$
\begin{gathered}
J(m, \emptyset) \geq \frac{\delta}{2}\|\Phi\|_{L^{2}\left(\mathrm{Q}_{0}\right)}^{2}, \\
\|\Phi\|_{L^{2}\left(\mathrm{Q}_{0}\right)} \longrightarrow \infty, \quad \text { as }\|m\|_{W(0, T ; V)} \longrightarrow \infty .
\end{gathered}
$$

Then,

$$
J(m, \varpi) \longrightarrow \infty, \quad \text { as }\|(m, \varpi)\|_{X} \longrightarrow \infty .
$$

Note that the norm is weakly lower semicontinuous [51]; we have that $J$ is weakly lower semicontinuous. Since $J(m, \varpi) \geq$ 0 , for all $(m, \emptyset) \in X$, holds, there exists $\kappa \geq 0$ defined by

$$
\kappa=\inf \{J(m, \omega) \mid(m, \varpi) \in X, e(m, \varpi)=0\},
$$

which means the existence of a minimizing sequence $\left\{\left(m^{n}\right.\right.$, $\left.\left.\omega^{n}\right)\right\}_{n \in \mathcal{N}}$ in $X$ such that

$$
\kappa=\lim _{n \rightarrow \infty} J\left(m^{n}, \omega^{n}\right), \quad e\left(m^{n}, \omega^{n}\right)=0, \quad \forall n \in \mathcal{N} .
$$

According to (62), there exists an element $\left(m^{*}, \varpi^{*}\right) \in X$ such that, when $n \rightarrow \infty$,

$$
\begin{gathered}
m^{n} \longrightarrow m^{*}, \quad \text { weakly, } m \in W(0, T ; V), \\
\omega^{n} \longrightarrow \omega^{*}, \quad \text { weakly, } \Phi \in L^{2}\left(Q_{0}\right) .
\end{gathered}
$$

From (65), it is easy to check that

$$
\lim _{n \rightarrow \infty} \int_{0}^{T}\left(m_{t}^{n}(t)-m_{t}^{*}(t), \phi(t)\right)_{V^{*}, V} d t=0,
$$

$$
\forall \phi \in L^{2}(0, T ; V)
$$

Thanks to the fact that $W(0, T ; V)$ is compactly embedded into $L^{2}\left(0, T ; L^{\infty}\right)$ [52] and $W(0, T ; V)$ is continuously embedded into $C(0, T ; H)$ [53], we obtain that $m^{n} \rightarrow m^{*}$ strongly in $L^{2}\left(0, T ; L^{\infty}\right)$ and $m^{n} \rightarrow m^{*}$ strongly in $C(0, T ; H)$. Furthermore, we have that $u^{n} \rightarrow u^{*}$ strongly in $C(0, T ; H)$.

Since the sequence $\left\{m^{n}\right\}_{n \in \mathcal{N}}$ converges weakly and $\left\|m^{n}\right\|_{W(0, T ; V)}$ is bounded [54], based on embedding theorem, we obtain that $\left\|m^{n}\right\|_{L^{2}\left(0, T ; L^{\infty}\right)}$ is bounded. In fact, $\left\|m^{*}\right\|_{L^{2}\left(0, T ; L^{\infty}\right)}$ is also bounded, because $m^{n} \rightarrow m^{*}$ strongly in $L^{2}\left(0, T ; L^{\infty}\right)$.

Thus, it follows from the Hölder inequality that

$$
\begin{aligned}
& \left|\int_{0}^{T} \int_{0}^{1}\left(u_{x}^{n} m^{n}-u_{x}^{*} m^{*}\right) \phi d x d t\right| \\
& =\left|\int_{0}^{T} \int_{0}^{1}\left(u_{x}^{n} m^{n}-u_{x}^{n} m^{*}+u_{x}^{n} m^{*}-u_{x}^{*} m^{*}\right) \phi d x d t\right| \\
& \leq\left|\int_{0}^{T} \int_{0}^{1} u_{x}^{n}\left(m^{n}-m^{*}\right) \phi d x d t\right| \\
& \quad+\left|\int_{0}^{T} \int_{0}^{1}\left(u_{x}^{n}-u_{x}^{*}\right) m^{*} \phi d x d t\right| \\
& \leq\left|\int_{0}^{T}\left\|m^{n}-m^{*}\right\|_{L^{\infty}}\left\|u^{n}\right\|_{H}\|\phi\|_{V} d t\right| \\
& \quad+\left|\int_{0}^{T}\left\|m^{*}\right\|_{L^{\infty}}\left\|u^{n}-u^{*}\right\|_{H}\|\phi\|_{V} d t\right| \\
& \leq\left\|m^{n}-m^{*}\right\|_{L^{2}\left(0, T ; L^{\infty}\right)}\left\|u^{n}\right\|_{C(0, T ; H)}\|\phi\|_{L^{2}(0, T ; V)} \\
& \quad+\left\|m^{*}\right\|_{L^{2}\left(0, T ; L^{\infty}\right)}\left\|u^{n}-u^{*}\right\|_{C(0, T ; H)}\|\phi\|_{L^{2}(0, T ; V)} \\
& \longrightarrow 0, \quad \forall \phi \in L^{2}(0, T ; V) .
\end{aligned}
$$

Similarly,

$$
\begin{aligned}
& \left|\int_{0}^{T} \int_{0}^{1}\left(u^{*} m^{*}-u^{n} m^{n}\right) \phi_{x} d x d t\right| \\
& =\left|\int_{0}^{T} \int_{0}^{1}\left(u^{*} m^{*}-u^{n} m^{*}+u^{n} m^{*}-u^{n} m^{n}\right) \phi_{x} d x d t\right| \\
& \leq\left|\int_{0}^{T} \int_{0}^{1}\left(u^{*} m^{*}-u^{n} m^{*}\right) \phi_{x} d x d t\right| \\
& \quad+\left|\int_{0}^{T} \int_{0}^{1}\left(u^{n} m^{*}-u^{n} m^{n}\right) \phi_{x} d x d t\right| \\
& \leq\left|\int_{0}^{T}\left\|m^{*}\right\|_{L^{\infty}}\left\|u^{*}-u^{n}\right\|_{H}\|\phi\|_{V} d t\right| \\
& \quad+\left|\int_{0}^{T}\left\|u^{n}\right\|_{H}\left\|m^{*}-m^{n}\right\|_{L^{\infty}}\|\phi\|_{V} d t\right|
\end{aligned}
$$




$$
\begin{aligned}
\leq & \left\|m^{*}\right\|_{L^{2}\left(0, T ; L^{\infty}\right)}\left\|u^{*}-u^{n}\right\|_{C(0, T ; H)}\|\phi\|_{L^{2}(0, T ; V)} \\
& +\left\|m^{*}-u^{n}\right\|_{L^{2}\left(0, T ; L^{\infty}\right)}\left\|u^{n}\right\|_{C(0, T ; H)}\|\phi\|_{L^{2}(0, T ; V)} \\
\longrightarrow & 0, \quad \forall \phi \in L^{2}(0, T ; V) .
\end{aligned}
$$

Then, we have

$$
\begin{aligned}
& \left|\int_{0}^{T} \int_{0}^{1}\left(u^{n} m_{x}^{n}-u^{*} m_{x}^{*}\right) \phi d x d t\right| \\
& =\mid \int_{0}^{T} \int_{0}^{1}\left(-u_{x}^{n} m^{n} \phi-u^{n} m^{n} \phi_{x}\right. \\
& \left.\quad+u_{x}^{*} m^{*} \phi+u^{*} m^{*} \phi_{x}\right) d x d t \mid \\
& \leq\left|\int_{0}^{T} \int_{0}^{1}\left(u_{x}^{*} m^{*}-u_{x}^{n} m^{n}\right) \phi d x d t\right| \\
& \quad+\left|\int_{0}^{T} \int_{0}^{1}\left(u^{*} m^{*}-u^{n} m^{n}\right) \phi_{x} d x d t\right| \\
& \longrightarrow 0, \quad \forall \phi \in L^{2}(0, T ; V) .
\end{aligned}
$$

By view of (66), we can obtain that

$$
\left|\int_{0}^{T} \int_{0}^{1}\left(B^{*} \omega^{n}-B^{*} \varpi^{*}\right) \phi d x d t\right| \longrightarrow 0, \quad \forall \phi \in L^{2}(0, T ; V) .
$$

According to the above discussion, we have that

$$
e_{1}\left(m^{*}, \varpi^{*}\right)=0, \quad \forall n \in \mathcal{N} .
$$

Thanks to the fact that $m^{*} \in W(0, T ; V)$ and $m^{n} \rightarrow m^{*}$ weakly in $W(0, T ; V)$, we have $m^{*}(0) \in H$ and $m^{n}(0) \rightarrow$ $m^{*}(0)$ weakly as $n \rightarrow \infty$. Furthermore, we obtain

$$
\left(m^{n}(0)-m^{*}(0), \phi\right) \longrightarrow 0, \quad n \longrightarrow \infty, \forall \phi \in H,
$$

which means that $e_{2}\left(m^{*}, \omega^{*}\right)=0$.

Therefore, we have that

$$
e\left(m^{*}, \omega^{*}\right)=0, \quad \text { in } Y .
$$

Thus, there exists an optimal solution $\left(m^{*}, \omega^{*}\right)$ to the problem (56). In the meantime, we can infer that there exists an optimal solution $\left(u^{*}, \omega^{*}\right)$ to the viscous shallow water equation due to the relation $u=\left(1-\partial_{x}^{2}\right)^{-1} m$. The proof of this theorem is completed.

\section{Acknowledgments}

The authors would like to thank all the anonymous referees for their careful reading and constructive comments. This work was supported by the Natural Science Foundation of China (nos. 11171135, 10420130638) and the Taizhou Social Development Project.

\section{References}

[1] D. D. Holm and M. F. Staley, "Nonlinear balance and exchange of stability of dynamics of solitons, peakons, ramps/cliffs and leftons in a 1+1 nonlinear evolutionary PDE," Physics Letters A, vol. 308, no. 5-6, pp. 437-444, 2003.

[2] H. R. Dullin, G. A. Gottwald, and D. D. Holm, "An integrable shallow water equation with linear and nonlinear dispersion," Physical Review Letters, vol. 87, pp. 4501-4504, 2001.

[3] A. Degasperis and M. Procesi, "Asymptotic integrability," in Symmetry and Perturbation Theory (Rome, 1998), A. Degasperis and G. Gaeta, Eds., pp. 23-37, World Scientific, River Edge, NJ, USA, 1999.

[4] R. Camassa and D. D. Holm, "An integrable shallow water equation with peaked solitons," Physical Review Letters, vol. 71, no. 11, pp. 1661-1664, 1993.

[5] A. Degasperis, D. D. Kholm, and A. N. I. Khon, "A new integrable equation with peakon solutions," Teoreticheskaya $i$ Matematicheskaya Fizika, vol. 133, no. 2, pp. 170-183, 2002.

[6] B. Fuchssteiner and A. S. Fokas, "Symplectic structures, their Bäcklund transformations and hereditary symmetries," Physica D, vol. 4, no. 1, pp. 47-66, 1981.

[7] B. Fuchssteiner, "Some tricks from the symmetry-toolbox for nonlinear equations: generalizations of the Camassa-Holm equation," Physica D, vol. 95, no. 3-4, pp. 229-243, 1996.

[8] A. Constantin, "On the scattering problem for the CamassaHolm equation," Proceedings of the Royal Society A, vol. 457, no. 2008, pp. 953-970, 2001.

[9] J. Lenells, "Traveling wave solutions of the Camassa-Holm equation," Journal of Differential Equations, vol. 217, no. 2, pp. 393-430, 2005.

[10] A. Constantin and W. A. Strauss, "Stability of the CamassaHolm solitons," Journal of Nonlinear Science, vol. 12, no. 4, pp. 415-422, 2002.

[11] A. Constantin and J. Escher, "Well-posedness, global existence, and blowup phenomena for a periodic quasi-linear hyperbolic equation," Communications on Pure and Applied Mathematics, vol. 51, no. 5, pp. 475-504, 1998.

[12] A. Constantin, "Existence of permanent and breaking waves for a shallow water equation: a geometric approach," Annales de l'Institut Fourier, vol. 50, no. 2, pp. 321-362, 2000.

[13] G. Rodríguez-Blanco, "On the Cauchy problem for the Camassa-Holm equation," Nonlinear Analysis: Theory, Methods \& Applications, vol. 46, no. 3, pp. 309-327, 2001.

[14] H. Dai, K.-H. Kwek, H. Gao, and C. Qu, "On the Cauchy problem of the Camassa-Holm equation," Frontiers of Mathematics in China, vol. 1, no. 1, pp. 144-159, 2006.

[15] A. Constantin and J. Escher, "Global existence and blow-up for a shallow water equation," Annali della Scuola Normale Superiore di Pisa. Classe di Scienze. Serie IV, vol. 26, no. 2, pp. 303-328, 1998.

[16] Y. A. Li and P. J. Olver, "Well-posedness and blow-up solutions for an integrable nonlinearly dispersive model wave equation," Journal of Differential Equations, vol. 162, no. 1, pp. 27-63, 2000.

[17] A. Bressan and A. Constantin, "Global conservative solutions of the Camassa-Holm equation," Archive for Rational Mechanics and Analysis, vol. 183, no. 2, pp. 215-239, 2007.

[18] Z. Xin and P. Zhang, "On the weak solutions to a shallow water equation," Communications on Pure and Applied Mathematics, vol. 53, no. 11, pp. 1411-1433, 2000. 
[19] A. Constantin and W. A. Strauss, "Stability of peakons," Communications on Pure and Applied Mathematics, vol. 53, no. 5, pp. 603-610, 2000.

[20] H. R. Dullin, G. A. Gottwald, and D. D. Holm, "Camassa-Holm, Korteweg-de Vries-5 and other asymptotically equivalent equations for shallow water waves," Fluid Dynamics Research, vol. 33, no. 1-2, pp. 73-95, 2003.

[21] D. D. Holm and M. F. Staley, "Wave structure and nonlinear balances in a family of evolutionary PDEs," SIAM Journal on Applied Dynamical Systems, vol. 2, no. 3, p. 323380, 2003.

[22] Y. Matsuno, "Multisoliton solutions of the Degasperis-Procesi equation and their peakon limit," Inverse Problems, vol. 21, no. 5, pp. 1553-1570, 2005.

[23] H. Lundmark and J. Szmigielski, "Multi-peakon solutions of the Degasperis-Procesi equation," Inverse Problems, vol. 19, no. 6, pp. 1241-1245, 2003.

[24] Z. Yin, "On the Cauchy problem for an integrable equation with peakon solutions," Illinois Journal of Mathematics, vol. 47, no. 3 , pp. 649-666, 2003.

[25] Z. Yin, "Global existence for a new periodic integrable equation," Journal of Mathematical Analysis and Applications, vol. 283, no. 1, pp. 129-139, 2003.

[26] Z. Yin, "Global solutions to a new integrable equation with peakons," Indiana University Mathematics Journal, vol. 53, no. 4, pp. 1189-1209, 2004.

[27] Z. Yin, "Global weak solutions for a new periodic integrable equation with peakon solutions," Journal of Functional Analysis, vol. 212, no. 1, pp. 182-194, 2004.

[28] Y. Liu and Z. Yin, "Global existence and blow-up phenomena for the Degasperis-Procesi equation," Communications in Mathematical Physics, vol. 267, no. 3, pp. 801-820, 2006.

[29] J. Escher, Y. Liu, and Z. Yin, "Global weak solutions and blowup structure for the Degasperis-Procesi equation," Journal of Functional Analysis, vol. 241, no. 2, pp. 457-485, 2006.

[30] J. Escher, Y. Liu, and Z. Yin, "Shock waves and blow-up phenomena for the periodic Degasperis-Procesi equation," Indiana University Mathematics Journal, vol. 56, no. 1, pp. 87-117, 2007.

[31] G. M. Coclite and K. H. Karlsen, "On the well-posedness of the Degasperis-Procesi equation," Journal of Functional Analysis, vol. 233, no. 1, pp. 60-91, 2006.

[32] S. Lai and Y. Wu, "Global solutions and blow-up phenomena to a shallow water equation," Journal of Differential Equations, vol. 249, no. 3, pp. 693-706, 2010.

[33] K. Ito and S. S. Ravindran, "A reduced basis method for control problems governed by PDEs," in Control and Estimation of Distributed Parameter Systems (Vorau, 1996), vol. 126 of International Series of Numerical Mathematics, pp. 153-168, Birkhäuser, Basel, Switzerland, 1998.

[34] J. A. Atwell and B. B. King, "Proper orthogonal decomposition for reduced basis feedback controllers for parabolic equations," Mathematical and Computer Modelling, vol. 33, no. 1-3, pp. 1-19, 2001.

[35] C. Hu and R. Temam, "Robust control of the KuramotoSivashinsky equation," Dynamics of Continuous, Discrete \& Impulsive Systems. Series B, vol. 8, no. 3, pp. 315-338, 2001.

[36] K. Kunisch and S. Volkwein, "Control of the Burgers equation by a reduced-order approach using proper orthogonal decomposition," Journal of Optimization Theory and Applications, vol. 102, no. 2, pp. 345-371, 1999.

[37] M. Hinze and S. Volkwein, "Analysis of instantaneous control for the Burgers equation," Nonlinear Analysis: Theory, Methods \& Applications, vol. 50, no. 1, pp. 1-26, 2002.
[38] R. Vedantham, "Optimal control of the viscous Burgers equation using an equivalent index method," Journal of Global Optimization, vol. 18, no. 3, pp. 255-263, 2000.

[39] B. Øksendal, "Optimal control of stochastic partial differential equations," Stochastic Analysis and Applications, vol. 23, no. 1, pp. 165-179, 2005.

[40] O. Ghattas and J. H. Bark, "Optimal control of two- and threedimensional incompressible Navier-Stokes flows," Journal of Computational Physics, vol. 136, pp. 231-244, 1997.

[41] J. E. Lagnese and G. Leugering, “Time-domain decomposition of optimal control problems for the wave equation," Systems \& Control Letters, vol. 48, no. 3-4, pp. 229-242, 2003.

[42] J. M. Yong, "Existence theory of optimal controls for distributed parameter systems," Kodai Mathematical Journal, vol. 15, no. 2, pp. 193-220, 1992.

[43] J. M. Yong and S. M. Zheng, "Feedback stabilization and optimal control for the Cahn-Hilliard equation," Nonlinear Analysis: Theory, Methods \& Applications, vol. 17, no. 5, pp. 431-444, 1991.

[44] S.-U. Ryu and A. Yagi, "Optimal control of Keller-Segel equations," Journal of Mathematical Analysis and Applications, vol. 256, no. 1, pp. 45-66, 2001.

[45] S.-U. Ryu, "Optimal control problems governed by some semilinear parabolic equations," Nonlinear Analysis: Theory, Methods \& Applications, vol. 56, no. 2, pp. 241-252, 2004.

[46] C. Shen, L. Tian, and A. Gao, "Optimal control of the viscous Dullin-Gottwalld-Holm equation," Nonlinear Analysis: Real World Applications, vol. 11, no. 1, pp. 480-491, 2010.

[47] L. Tian and C. Shen, "Optimal control of the viscous Degasperis-Procesi equation," Journal of Mathematical Physics, vol. 48, no. 11, Article ID 113513, 16 pages, 2007.

[48] L. Tian, C. Shen, and D. Ding, "Optimal control of the viscous Camassa-Holm equation," Nonlinear Analysis: Real World Applications, vol. 10, no. 1, pp. 519-530, 2009.

[49] X. Zhao and C. Liu, "Optimal control problem for viscous Cahn-Hilliard equation," Nonlinear Analysis: Theory, Methods \& Applications, vol. 74, no. 17, pp. 6348-6357, 2011.

[50] J.-L. Lions, Optimal Control of Systems Governed by Partial Differential Equations, Springer, New York, NY, USA, 1971.

[51] A. Wouk, A Course of Applied Functional Analysis, Pure and Applied Mathematics, Wiley-Interscience, New York, NY, USA, 1979.

[52] R. Temam, Navier-Stokes Equations: Theory and Numerical Analysis, vol. 2 of Studies in Mathematics and Its Applications, North-Holland, Amsterdam, The Netherlands, 1979.

[53] R. Dautray and J.-L. Lions, Mathematical Analysis and Numerical Methods for Science and Technology. Volume 5: Evolution Problems I, Springer, Berlin, Germany, 1992.

[54] E. Kreyszig, Introductory Functional Analysis with Applications, John Wiley \& Sons, New York, NY, USA, 1978. 


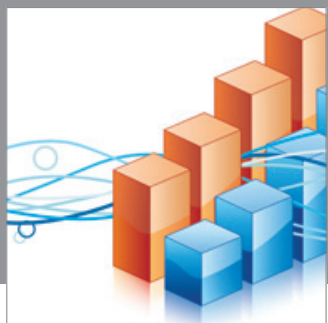

Advances in

Operations Research

mansans

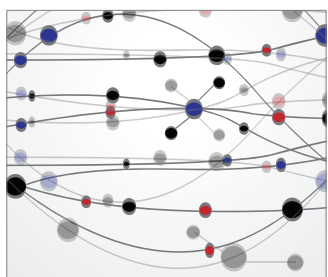

The Scientific World Journal
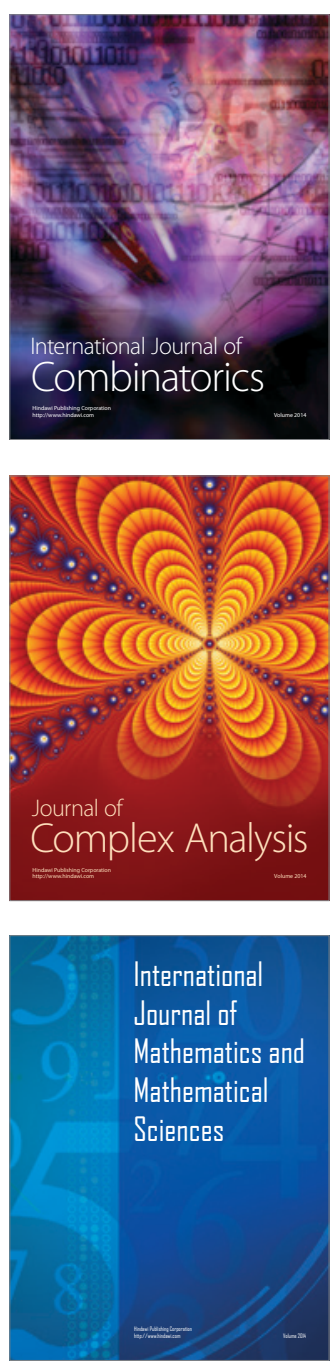
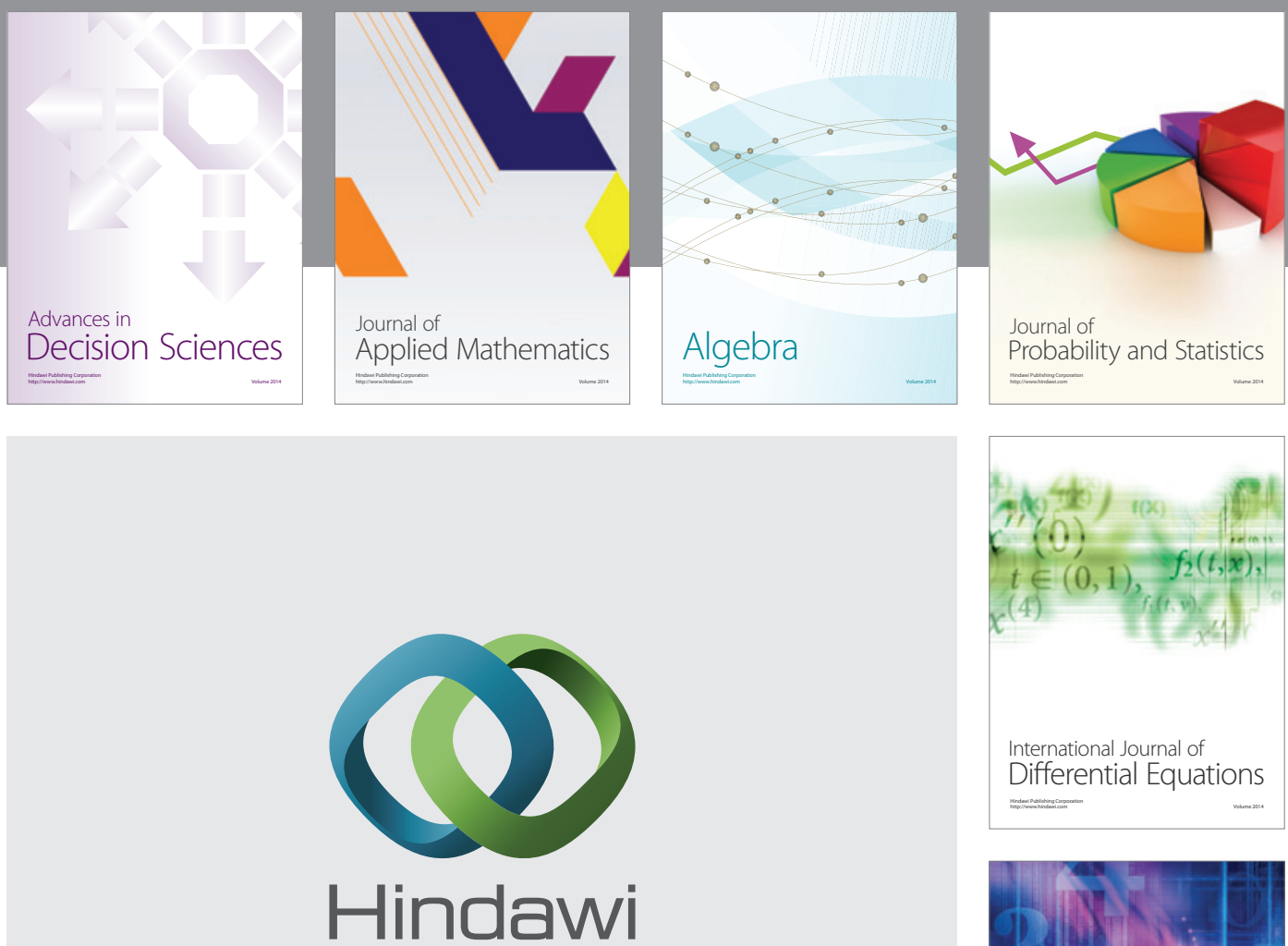

Submit your manuscripts at http://www.hindawi.com
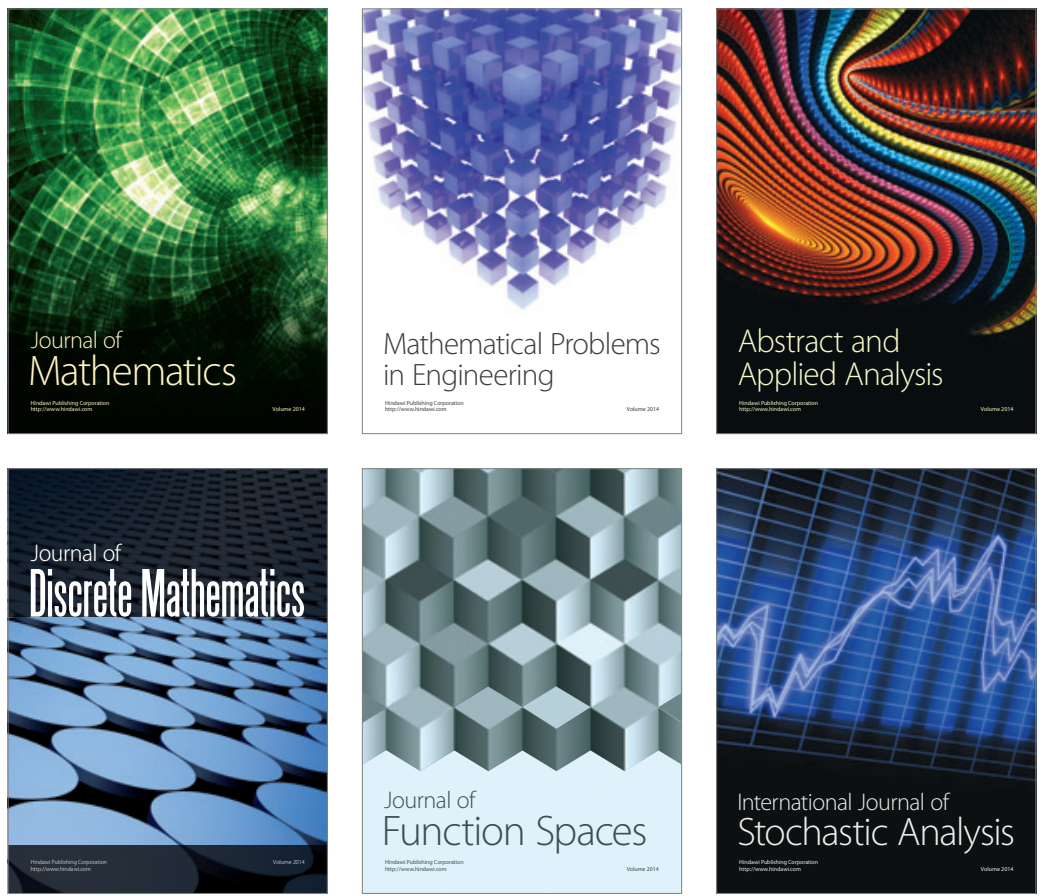

Journal of

Function Spaces

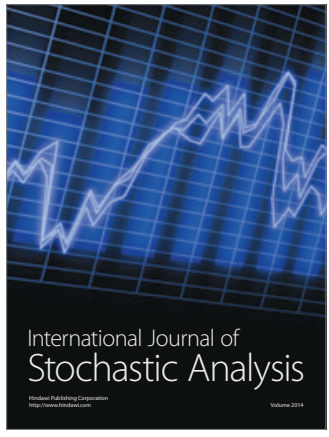

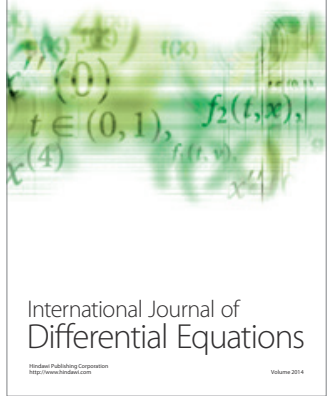
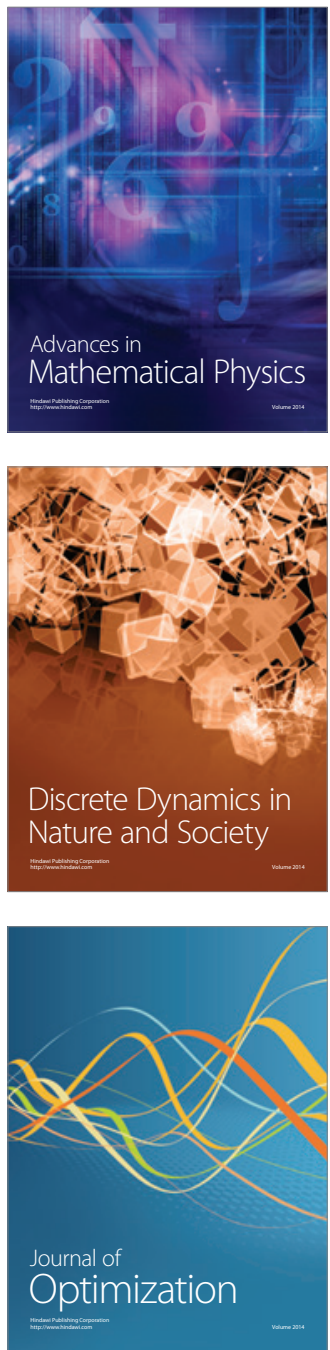